Check for updates

Cite this: J. Mater. Chem. C, 2017, 5,5824

Received 16th January 2017, Accepted 8th May 2017

DOI: $10.1039 / \mathrm{ctc00257b}$

rsc.li/materials-c

\title{
Infrared electrochromic conducting polymer devices $\dagger$
}

\author{
Robert Brooke, ${ }^{a}$ Evangelia Mitraka, ${ }^{a}$ Samim Sardar, ${ }^{a}$ Mats Sandberg, (D) ab \\ Anurak Sawatdee, ${ }^{b}$ Magnus Berggren, ${ }^{a}$ Xavier Crispin (D) ${ }^{a}$ and \\ Magnus P. Jonsson (D) *a
}

\begin{abstract}
The conducting polymer poly(3,4-ethylenedioxythiophene) (PEDOT) is well known for its electrochromic properties in the visible region. Less focus has been devoted to the infrared (IR) wavelength range, although tunable IR properties could enable a wide range of novel applications. As an example, modern day vehicles have thermal cameras to identify pedestrians and animals in total darkness, but road and speed signs cannot be easily visualized by these imaging systems. IR electrochromism could enable a new generation of dynamic road signs that are compatible with thermal imaging, while simultaneously providing contrast also in the visible region. Here, we present the first metal-free flexible IR electrochromic devices, based on PEDOT:Tosylate as both the electrochromic material and electrodes. Lateral electrochromic devices enabled a detailed investigation of the IR electrochromism of thin PEDOT:Tosylate films, revealing large changes in their thermal signature, with effective temperature changes up to $10{ }^{\circ} \mathrm{C}$ between the oxidized $(1.5 \mathrm{~V})$ and reduced $(-1.5 \mathrm{~V})$ states of the polymer. Larger scale $(7 \times 7 \mathrm{~cm})$ vertical electrochromic devices demonstrate practical suitability and showed effective temperature changes of approximately $7{ }^{\circ} \mathrm{C}$, with good optical memory and fast switching $(1.9 \mathrm{~s}$ from the oxidized state to the reduced state and $3.3 \mathrm{~s}$ for the reversed switching). The results are highly encouraging for using PEDOT:Tosylate for IR electrochromic applications.
\end{abstract}

\section{Introduction}

Thermal imaging cameras and displays in modern automobiles are designed to enhance the safety of driving in total darkness and difficult weather conditions such as rain or smoke. ${ }^{1}$ The development of thermal sensing in vehicles aids the user to easily identify pedestrians and animals. ${ }^{2,3}$ Both active and passive thermal sensory systems exist ${ }^{4,5}$ and both rely on differences in IR reflectivity (and hence emissivity) between the environment and objects such as pedestrians or animals. However, both systems have issues discerning road and street signs, which has been discussed as a potential problem for drivers in difficult driving conditions. ${ }^{6}$ For active thermal systems, road signs are too reflective and not only blur their information, but may also disrupt or hide other entities within the display. ${ }^{7}$ Road and speed signs also present a problem to passive systems, as they possess no temperature difference compared to the surrounding environment, making them effectively invisible in the display. ${ }^{8}$

\footnotetext{
${ }^{a}$ Linköping University, Department of Science and Technology,

Laboratory for Organic Electronics, SE-601 74 Norrköping, Sweden.

E-mail: magnus.jonsson@liu.se

${ }^{b}$ Acreo Swedish ICT, SE-601 74 Norrköping, Sweden

$\dagger$ Electronic supplementary information (ESI) available. See DOI: 10.1039/c7tc00257b
}

IR electrochromic devices may provide an answer to increasing the visibility of speed signs, stop signs and other visual information to thermal cameras, by modulating the infrared signature and effective temperature of the materials.

Electrochromism refers to electrically triggered changes in the optical properties of a material. ${ }^{9}$ Since the discovery of electrochromism in metal oxide thin films, advances in materials, processes and device designs have followed. ${ }^{9-12}$ The use of conductive polymers (CPs) as electrochromic materials is particularly promising, with advantages such as flexibility, ease of processing and ability to manipulate their chemistry for a wide range of optical properties. ${ }^{13,14}$

The CP poly(3,4-ethylenedioxythiophene) (PEDOT) has been studied extensively based on its high conductivity, air stability and electrochromic behavior. ${ }^{15}$ PEDOT exhibits a high optical density blue color in its reduced state and a low optical density transparent blue color in its oxidized state. This makes it an excellent candidate for electrochromic applications in the visible wavelength region, such as smart windows ${ }^{16,17}$ for buildings and the automotive industry. ${ }^{17,18}$ Comparatively, electrochromism of PEDOT in the IR wavelength range has been only sparsely reported. ${ }^{19-23}$ While most reports on the electrochromism of PEDOT were limited to the visible and near IR regions, ${ }^{16,23}$ many applications (road signs, safety clothing, space craft, etc.) 
could take advantage of an organic emissive modulator in the IR region. As an example, a recent study ${ }^{20}$ presented an electrochromic PEDOT device aiming at camouflaging a heated panel behind the device, and showed modulation within the IR region corresponding to a few degrees centigrade. Another study reported Fourier transform IR (FT-IR) reflectance of PEDOT. ${ }^{19}$ Both examples employed inorganic metal electrodes (ITO or gold) to facilitate the switching of the devices. Other CPs like polyaniline blends have also been investigated for IR applications, showing high variation in emissivity and good electrochromic characteristics. ${ }^{24}$ However, due to the relatively low conductivity of the polyaniline blends, they could not be used as electrodes in addition to acting as active electrochromic materials. In turn, this means that additional electrodes composed of metal or metal oxides are required, which reduce flexibility and complicate device design. More established inorganic electrochromic materials such as tungsten oxide have also been investigated for use in the IR, with reflectance or emissivity variations of approximately $30 \% .^{25}$

Here we report encapsulated metal-free IR electrochromic devices, employing PEDOT:Tosylate (PEDOT:Tos) not only as the electrochromic material, but also as the electrodes. This was possible owing to the high conductivity of our PEDOT:Tos films, as produced by vapor phase polymerization (VPP). VPP is a synthesis technique with the potential of providing conductivities that can rival that of transparent metal oxide electrodes such as ITO. $^{26-28}$ The method can produce PEDOT:Tos films with conductivities far above $1000 \mathrm{~S} \mathrm{~cm}^{-128,29}$ and values up to approximately $7600 \mathrm{~S} \mathrm{~cm}^{-1}$ have been reported for single crystal PEDOT nanowires. ${ }^{30}$ Additional advantages of VPP include simple introduction of additives, the ability for patterning, scalability, simple experimental design, and flexibility with respect to the substrate material. ${ }^{31-34}$ Here we demonstrate the possibility of using polyethylene (PE) as the substrate, which is transparent in the IR region, ${ }^{35}$ while also being lightweight and flexible.

Our study is based on both lateral and vertical IR electrochromic PEDOT:Tos devices. Lateral devices enabled a detailed investigation of the IR electrochromic properties of the PEDOT:Tos upon switching between its oxidized and reduced states. Thermal imaging complements the spectroscopic FT-IR analysis and reveals large effective temperature changes (approximately $10{ }^{\circ} \mathrm{C}$ ) of the films. We also designed and investigated vertical IR electrochromic PEDOT:Tos devices, which will be preferable for most practical applications. Thermal imaging confirms that also these devices provide large (approximately $7{ }^{\circ} \mathrm{C}$ ) effective temperature changes upon electrochromic switching. Based on these promising results, we performed a more detailed study of vertical IR electrochromic PEDOT:Tos devices, showing also rapid switching (few seconds) and good optical memory over a period of at least 10 hours.

\section{Experimental}

\section{Chemicals}

3,4-Ethylenedioxythiophene, 1-ethyl-3-methylimidazolium ethyl sulfate (EMIM-ES), ethanol, poly(ethylene glycol)-poly(propylene glycol)-poly(ethylene glycol) (PEG-PPG-PEG, $5800 M_{\mathrm{w}}$ ), and hydroxyl ethyl cellulose were purchased from Sigma Aldrich. Iron tosylate (Clevios CB-40, $40 \mathrm{wt} \%$ in butanol) was obtained from Heraeus. All chemicals were used as received.

\section{Device fabrication}

PEDOT:Tos films were synthesized using VPP, as previously described in detail. ${ }^{36}$ The oxidant solution was composed of $\mathrm{Fe}(\mathrm{Tos})_{3}(12.3 \mathrm{wt} \%)$ and PEG-PPG-PEG (23.1 wt\%) dissolved in ethanol. PE substrates (up to $7 \times 7 \mathrm{~cm}$ ) were washed in ethanol and placed on supporting glass to allow oxidant deposition. The oxidant was spin coated onto the PE substrates at $1500 \mathrm{rpm}$ for $30 \mathrm{~s}$ followed by placing the substrate in a VPP chamber. The chamber was heated to $50{ }^{\circ} \mathrm{C}$ and operated at a pressure of $-70 \mathrm{cmHg}$. The polymerization was allowed to continue for $180 \mathrm{~min}$, which correlated with an average PEDOT:Tos thickness of approximately $170 \mathrm{~nm}$. The exception was for larger scale vertical devices, for which the PEDOT:Tos was synthesized overnight to ensure that no defects were present in the thin film. The films were then gently washed with ethanol to remove excess oxidant and unreacted EDOT monomers. We note that heating steps were avoided in the procedure due to the low melting point $\left(135^{\circ} \mathrm{C}\right)^{37}$ of the PE substrates. Encapsulation of the devices was achieved using double-sided adhesive polyethylene terephthalate (PET). To avoid short circuits, we used a gel-like electrolyte instead of a liquid-based electrolyte. The gel was based on the ionic liquid EMIM-ES with hydroxyl ethyl cellulose $(7.5 \mathrm{wt} \%)$ as the gelling agent. ${ }^{38}$ The ionic liquid becomes gel-like (highly viscous ionic liquid). For the lateral devices the electrolyte was applied and spread manually before encapsulation. For vertical devices, a polypropylene separator soaked in the gel electrolyte was incorporated into the design to further avoid electrical short circuits.

\section{Spectroscopic and thermal characterization}

IR transmittance and reflectance spectra of lateral PEDOT:Tos electrochromic devices were obtained by Fourier transform infrared spectroscopy (FT-IR Equinox 55 and model Tensor 27, Bruker) and analyzed using a commercial software (Opus). Conversion of FT-IR reflectance to emissivity (for opaque samples) was calculated using:

$$
\varepsilon=1-R
$$

where $\varepsilon$ is the emissivity and $R$ is the reflectance. ${ }^{39}$ Transmittance spectra from the visible to the near IR were acquired using a UV/Vis/NIR spectrometer (Lambda 900, PerkinElmer). Control transmittance spectra for blank PE substrates and gel ionic liquid encapsulated by PE substrates can be found in the $\mathrm{ESI} \dagger$ (Fig. S1).

Thermal images were captured using a ThermaCAM ${ }^{\mathrm{TM}}$ SC640 with a spectral range of 7.5-13 $\mu \mathrm{m}$ and analyzed using a commercial software (FLIR systems ThermaCAM Researcher). The images were captured outside on a clear day to ensure that the reflection of the sky gave an appropriate scale for the measurements. A highly emissive metal surface and a less emissive rubber 'black body' were used as reference materials which allowed for an 
effective temperature range of approximately -44 to $8{ }^{\circ} \mathrm{C}$ (reference material can be seen in Fig. S2, ESI $\dagger$ ). We note that the effective temperature relates to the temperature as viewed by the IR thermal camera (based on changes in the emissivity), rather than actual temperature changes related to the electrochromic devices becoming warmer or colder.

The conductivity of the polymer films were measured using the four-point probe method, using evaporated gold probes on glass substrates. The conductivity $\sigma$ of the PEDOT:Tos thin films were calculated using:

$$
\sigma=\frac{L}{r A}
$$

where $r$ is the measured resistance, and $L$ and $A$ are the length and cross-sectional area of the probed region, respectively (SI units).

Energy consumption upon electrochromic switching behavior was evaluated using chronoamperometry (Autolab type III). After an initial $10 \mathrm{~s}$ equilibrium period at $0 \mathrm{~V}$, the devices were switched back and forth 10 times by repeatedly applying $1.5 \mathrm{~V}$ for 1 minute and $-1.5 \mathrm{~V}$ for $1 \mathrm{~min}$. This potential range was used throughout the work, and chosen based on the fact that the majority of the oxidation and reduction occurs within these voltage limits, and because previous reports have shown little to no degradation of PEDOT:Tos films while operating at these potentials. ${ }^{18}$ The charge $C_{\text {switch }}$ corresponding to one switch was calculated by time-integration of the measured current for each switch and the energy consumption per active area for each switch was calculated using:

$$
E_{\text {switch }}=\frac{C_{\text {switch }} \times V}{A}
$$

where $E_{\text {switch }}$ is the energy, $V$ is the applied voltage and $A$ is the active area of the device.

Normalized reflectance data for switching and optical memory experiments were obtained by illuminating the devices using a $100 \mathrm{~W}$ xenon lamp and collecting reflected light with a light guide connected to a spectrograph (Andor Shamrock 303i, with a Newton CCD detector). A separate power supply was used to switch the electrochromic devices. The optical memory experiments were performed using the same procedure, but with the device left disconnected from the power supply while measuring reflected light over time.

\section{Results and discussion}

Fig. 1 shows the device designs of the lateral (a) and vertical (b) electrochromic devices. The lateral devices enable side-byside investigation of the oxidized and reduced states of the PEDOT:Tos electrode and is also suitable for transmittance spectroscopy, as the probe beam penetrates only one of the two electrodes. The lateral geometry also practically eliminates the risk of electrical short circuit between the electrodes. By contrast, vertical devices require a separator to avoid short circuits. Fig. 1c and d show photographs of a typical lateral PEDOT:Tos electrochromic device, demonstrating high flexibility of the system and high optical contrast between the two polymer
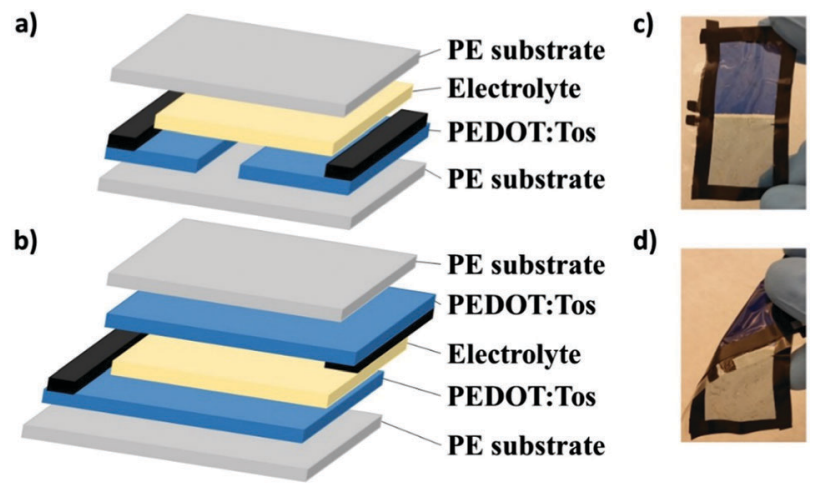

Fig. 1 (a) Lateral electrochromic device architecture, (b) vertical electrochromic device architecture. The black regions in (a) and (b) represent the electrical contacts to the PEDOT:Tos films. (c) Image of a lateral device, showing high contrast in the visible region for the PEDOT:Tos electrodes. (d) Image demonstrating the high flexibility of the same electrochromic device.

states. The dark blue regions in Fig. $1 \mathrm{c}$ and $\mathrm{d}$ correspond to the reduced deep blue state of the PEDOT:Tos, while the more transparent light blue region corresponds to the oxidized state. From these optical images it is evident that these devices, including the use of the non-conventional PE substrate, provide good contrast in the visible region, to complement the IR electrochromism studied in detail below.

Both the lateral and the vertical designs use the PEDOT:Tos films as electrodes to control the electrochemical switching. This reduces the number of required layers compared with previous concepts employing additional metal electrode layers. ${ }^{19,20}$ In turn, this enables more cost-effective and faster device production. This strategy requires that the polymer films remain conducting during device operation. Four-probe measurements show that the conductivity of the pristine PEDOT:Tos films was approximately $1000 \mathrm{~S} \mathrm{~cm}^{-1}$, which is more than sufficient for use as electrodes. Since it was not possible to measure the in situ conductivity of the oxidized and reduced PEDOT:Tos films of the devices, we instead directly test their function as electrodes by switching the devices. Both lateral and vertical electrochromic devices could be successfully and repeatedly switched, proving that both the oxidized (high conducting) and the reduced (less conducting) PEDOT:Tos films are sufficiently conducting to act as electrodes and facilitate switching.

The switching time of the lateral electrochromic devices was on the order of a few minutes (see Fig. S3, ESI $\dagger$ ). This relatively slow switching speed can be attributed to the large size $(\approx 3 \times 7 \mathrm{~cm})$ of the devices combined with the lateral geometry where only the edges of the two electrochromic electrodes are in close proximity. The use of a highly viscous electrolyte may also lead to lower switching speeds compared with the use of liquid alternatives, but with the added benefit of avoiding short circuits and preventing leakage. We note also that the lateral devices were primarily devised to allow the investigation of the individual PEDOT layers, while we further below evaluate the potential of the concept for practical IR electrochromic applications based on a detailed investigation of switching speed and other performance parameters for vertical devices. 

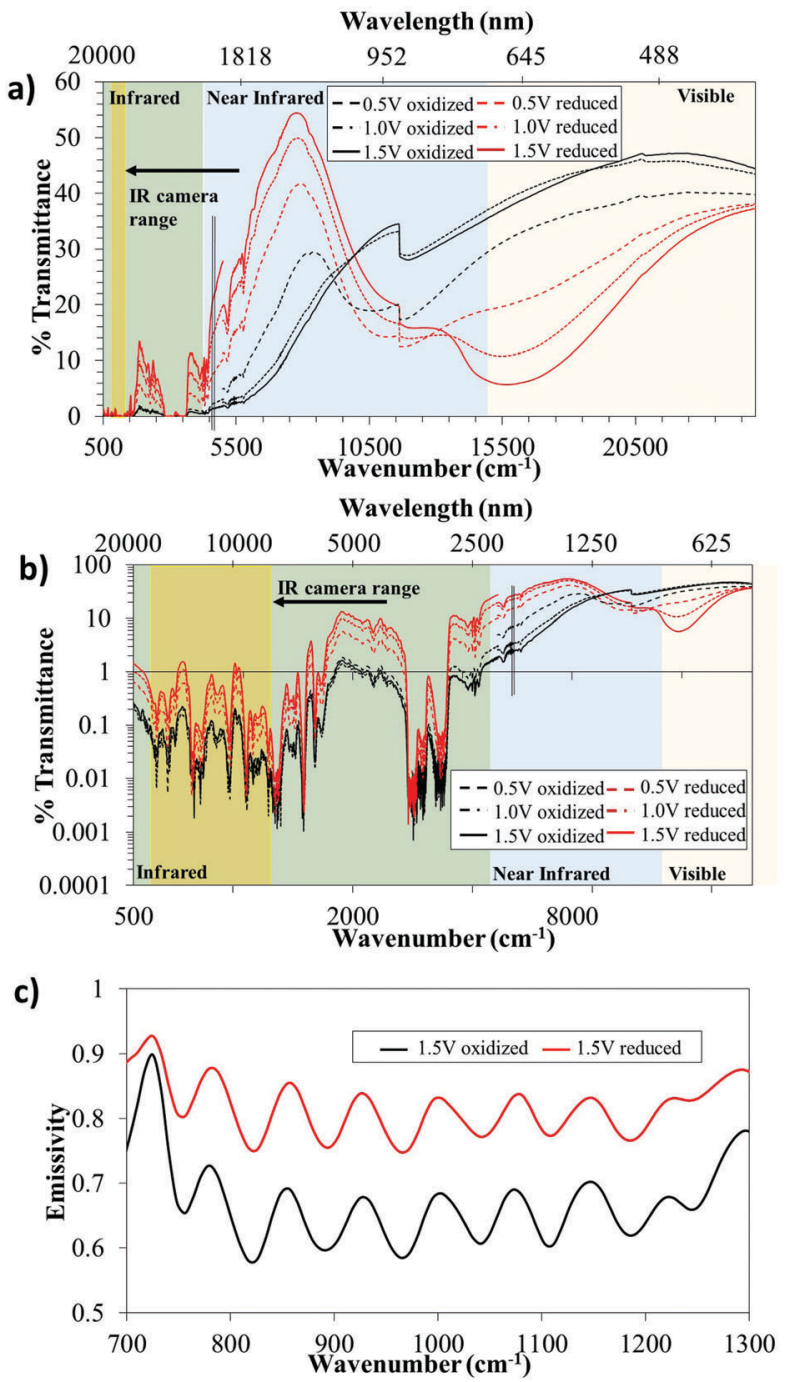

Fig. 2 Transmittance of a PEDOT:Tos lateral electrochromic device presented in a linear scale (a) and in a logarithmic scale (b), based on combined FT-IR and UV-vis spectra to show the whole range from visible to IR regions. (c) IR emissivity of oxidized and reduced PEDOT:Tos films of a lateral device, as measured using reflectance FT-IR.

Fig. 2 presents the electrochromic behavior (from the visible to the far IR region) for a lateral PEDOT:Tos device. Transmittance spectra were obtained in situ for the device using both FTIR and UV-vis transmittance spectroscopy. We combined the wavelength regions into one graph to provide a large-range perspective of the electrochromic behavior, and present the results in linear and logarithmic scales in Fig. 2a and b, respectively. We first note that there are significant spectroscopic differences between the oxidized (black) and reduced (red) polymer states throughout the full wavelength range. In addition, these differences could be tuned by varying the magnitude of the applied voltage. These results imply that the system is suitable for electrochromic applications extending from visible to far into the IR region. The oxidized states of the PEDOT:Tos film (black lines in Fig. 2) show high transmittance in the visible region, while light is strongly absorbed and/or reflected (low transmittance) in the whole IR wavelength range. This indicates that the polymer electrode contains free electrons and that it has no band gap. These findings are in agreement with previous work, including recent reports on semimetallic behavior of PEDOT:Tos films. ${ }^{31}$ Switching the PEDOT:Tos film to its reduced state (red lines in Fig. 2) reduces the free charge carrier absorption in the IR, while the well-known electrochromic blue color of PEDOT arises as a transmittance dip at approximately $600 \mathrm{~nm}$.

To evaluate the use of the observed IR electrochromism in applications related to thermal imaging, we turn to the range of interest for the thermal IR camera $(7.5-13 \mu \mathrm{m})$. The shaded yellow regions in Fig. 2a-c indicate this range. We note that the transmittance is very low $(<1 \%)$ in this region for both states of the device, although switching still provides modulation of this low transmittance. The low transmittance is primarily attributed to the electrolyte, which is known for its large electrochemical window and to enable stable switching of PEDOT:Tos devices, ${ }^{40}$ but provides low transmittance in the IR region (see Fig. S1, ESI $\dagger$ ). Hence, transmission-based applications may benefit from reducing the electrolyte and/or the polymer thicknesses, or from exploring alternative electrolytes. However, we note that the targeted practical applications, including IR-compatible road signs, will not be based on transmittance, but on reflectionmode measurements and modulation of the emissivity of the devices. In turn, emissivity modulation for vertical devices (see Fig. 1a) can benefit from electrolytes with low IR transmission, since this will prevent interfering signals from the bottom PEDOT:Tos electrode.

Fig. 2c shows the emissivity for a lateral device for the PEDOT:Tos in the oxidized (black) and reduced (red) states (approximating the sample as opaque, based on the low transmittance in this spectral region). It is clear that there are significant changes in the emissivity throughout the wavelength range of the thermal IR camera, with an averaged emissivity increase upon polymer reduction of approximately $15.3 \%$ (average value based on three different devices). As a comparison, the state-of-the art metal oxide active components were reported to provide around 30\% modulation. ${ }^{25}$ We judge this to be promising for the presented concept, in particular considering that there is room for further optimization of our proof-of-concept devices.

The strong modulation of the PEDOT:Tos emissivity in the IR region encouraged a more direct investigation of the thermal imaging possibilities of the electrochromic devices. We first present results for lateral devices, which allows the oxidized and reduced states of the PEDOT:Tos electrodes to be viewed simultaneously side by side. As seen in Fig. 3a, the initial reduced PEDOT:Tos shows an effective temperature of $-0.9{ }^{\circ} \mathrm{C}$ and the effective temperature drops to $-10.6{ }^{\circ} \mathrm{C}$ for the oxidized PEDOT:Tos. This almost $10{ }^{\circ} \mathrm{C}$ modulation of the effective temperature constitutes an improvement of almost one order of magnitude compared with previous PEDOT-based IR electrochromic devices. ${ }^{20}$ The decrease in the effective temperature of the PEDOT:Tos upon oxidation is in agreement with the lower emissivity for the oxidized state (see Fig. 2c).

Next, we investigate the IR electrochromism and thermal contrast for vertical electrochromic devices, which will be 
a)
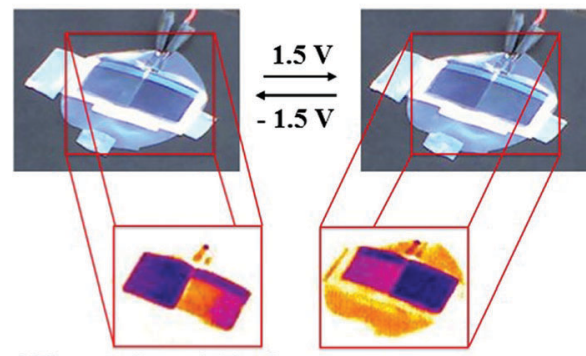

Lateral Electrochromic Device

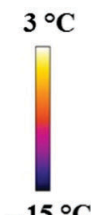

b)
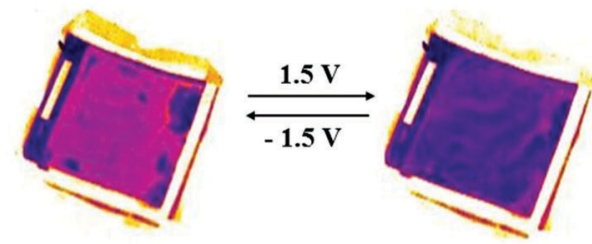

$3^{\circ} \mathrm{C}$

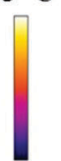

Vertical Electrochromic Device

$-15^{\circ} \mathrm{C}$

Fig. 3 (a) Visible (top) and thermal images (bottom) of a lateral PEDOT: Tos electrochromic device. A voltage of $1.5 \mathrm{~V}$ was applied to the electrochromic device and then reversed to show that both electroactive regions undergo oxidation and reduction. The temperature change from one state to the next was $9.7^{\circ} \mathrm{C}\left(-0.9\right.$ to $-10.6{ }^{\circ} \mathrm{C}$, as determined from analysis of the thermal images). (b) Vertical electrochromic PEDOT:Tos device in its reduced (left) and oxidized (right) states (referring to the PEDOT:Tos electrode facing towards the thermal camera), giving an effective temperature modulation of $6.3^{\circ} \mathrm{C}\left(-8.9\right.$ to $\left.-3.3^{\circ} \mathrm{C}\right)$.

preferable in most practical applications since they present only one state at a time. Fig. $3 \mathrm{~b}$ shows thermal images of a vertical device in its oxidized (right) and reduced (left) states. It is clear that strong modulation can be achieved also for vertical devices. We observe an effective temperature modulation of $6.3{ }^{\circ} \mathrm{C}$ between the oxidized and reduced states. The lower effective temperature modulation for the vertical device compared to the lateral device may be due to the additional layers within the vertical device, and that materials below the top PEDOT:Tos film may contribute to the total IR signature. Importantly, the modulations for both vertical and lateral devices are much higher than those for previously reported PEDOT electrochromic devices. ${ }^{20}$ This improved thermal modulation of our devices may be related to the higher conductivity or higher electron mobility of the PEDOT:Tos used in this work, and the corresponding simplified electrochromic device design without additional metal electrodes that may interfere with the modulation. Our results comprise encouraging steps for using PEDOT:Tos in practical IR electrochromic applications.

Next, we evaluate the vertical IR electrochromic PEDOT:Tos devices for practical applications by investigating their performance with respect to power consumption, optical memory, switching speed and stability. Chronoamperometry was used to measure power consumption and the amount of charge transferred during each switch. Fig. 4a shows the current $v$ s. time (black) during multiple switching steps for a vertical device. Based on the calculated charge and the applied voltage we calculate the energy per effective electrode area for each switch to only $16.5 \mathrm{~J} \mathrm{~m}^{-2}$ (Table S1, ESI $\dagger$ ). With respect to power consumption, we also note that PEDOT:Tos typically has a good optical memory, in the sense that it can remain in a switched state also after disconnecting the power supply. ${ }^{16}$ This allows for a lower total power consumption compared to inorganic-based systems that require continuous power to maintain their states. ${ }^{12,41}$

Fig. $4 \mathrm{~b}$ presents the results for the optical memory of a vertical PEDOT:Tos electrochromic device. For the reduced 'on' state (black), the device first shows a moderate relaxation within the first hour after disconnecting the power supply, after which it remains in a reduced state for at least 10 hours. If instead disconnected from the oxidized 'off' state, the device also shows good optical memory. After a relaxation period of about 4 hours, the device stabilizes close to the neutral state of the PEDOT:Tos. Importantly, the two relaxed 'on' and 'off' states are clearly separated from each other also after 10 hours, which means that the device will provide contrast between 'on' and 'off states for long periods after removing the power supply. For applications where it is important to maintain the devices at the initial reduced or oxidized states, the good optical memory could be complemented by short low-power electrical pulses. To confirm that the good optical memory applies to the IR region, we performed a secondary investigation of the IR transmittance of a lateral PEDOT:Tos electrochromic device, as presented in Fig. S4 (ESI $\dagger$ ). Indeed, only very small relaxation towards the neutral state could be observed 4 hours after disconnecting a fully reduced device. These results prove that the PEDOT:Tos electrochromic devices can maintain their IR signature hours after the initial electrochemical switching.

Next, we turn to the switching speed of the vertical devices. Fig. 4c and Fig. S5 (ESI $\dagger$ ) present the switching behavior and show that switching from the oxidized state to the reduced state takes only around $1.9 \mathrm{~s}$ (quantified based on the decrease from $90 \%$ to $10 \%$ of the normalized reflectance) while the switching back from the reduced state to the oxidized state took approximately $3.3 \mathrm{~s}$ (quantified based on the increase from $10 \%$ to $90 \%$ of the normalized reflectance). Hence, while the amplitude of the thermal modulation is somewhat lower for the vertical design compared with the lateral design, the switching speed is improved by around two orders of magnitudes. In turn, this rapid switching makes the IR electrochromic devices suitable for many applications, including for road signs with improved visibility. The longer switching time from the reduced state to the oxidized state compared with the opposite direction is consistent with previous reports, which attributed this effect to differences in the charge movement for the different redox reactions. ${ }^{42,43}$

Finally, we use cyclic voltammetry to investigate the stability upon repeated switching for a vertical device. The results are presented in Fig. 4d and show that the devices perform well within the first 100 cycles, with only small changes. While significant degradation was observed after 1000 cycles, we note that these results are based on proof of concept devices and there is a possibility to further optimize the device design for improved shelf life and cycle stability. We believe that the stability is encouraging at this stage, especially as the good optical memory allows the devices to be used for long times with only infrequent exposure to applied voltages to maintain the desired state of the 
a)

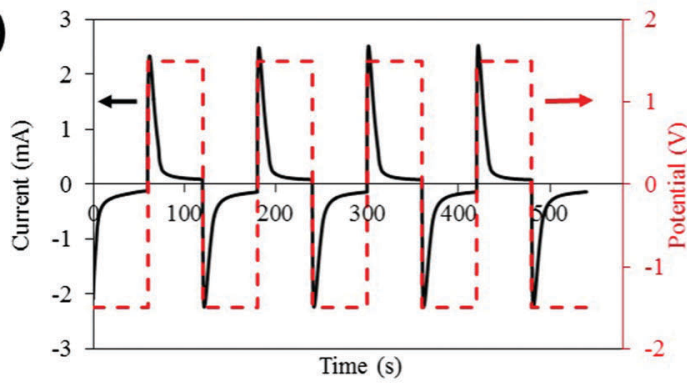

c)

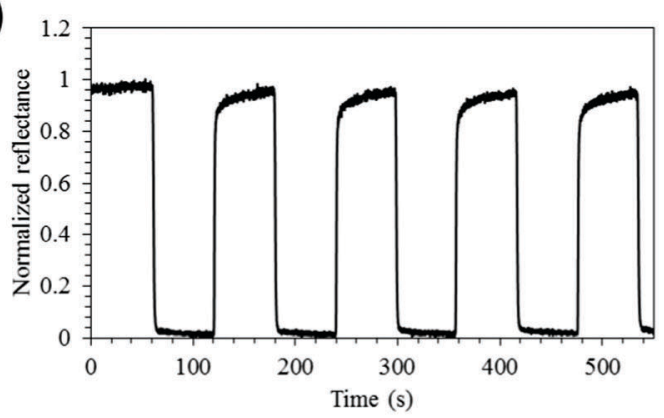

b)

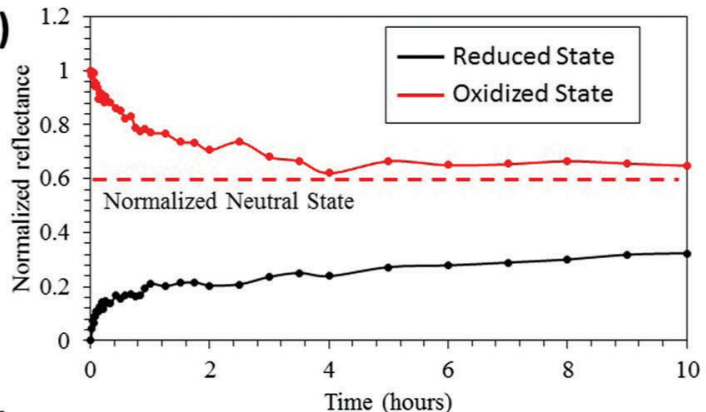

d)

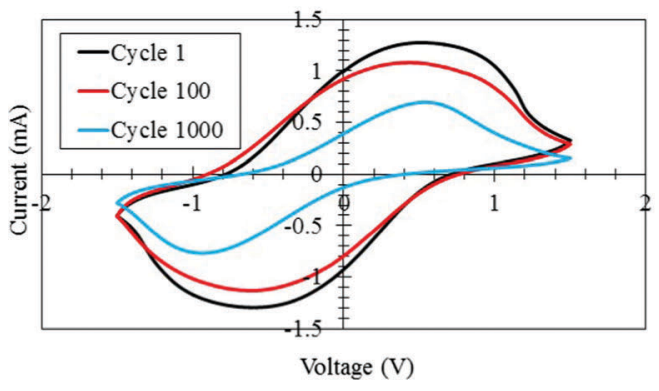

Fig. 4 Electrochromic characterization of a vertical PEDOT:Tos electrochromic device. (a) Chronoamperometry for a vertical PEDOT:Tos electrochromic device, with current in black and the applied voltage in dashed red. Conditioning potential ( $0 \mathrm{~V}$ ) was applied for $10 \mathrm{~s}$ in order to bring the devices in the neutral state, followed by cycles of $1.5 \mathrm{~V}$ for $60 \mathrm{~s}$ and $-1.5 \mathrm{~V}$ for $60 \mathrm{~s}$. (b) Optical memory investigation for the oxidized (red) and reduced (black) states of a vertical electrochromic PEDOT:Tos device. The optical memory was measured through variations in reflectance at $600 \mathrm{~nm}$ and presented normalized to the values for the fully reduced state (0) and the fully oxidized state (1). The neutral state (0 V) is marked with a red dashed line. (c) Switching speed investigation where the device was exposed to cycles of 1.5 and $-1.5 \mathrm{~V}$, presenting the normalized reflectance measured at $600 \mathrm{~nm}$ wavelength over time. (d) Cyclic voltammetry stability investigation, showing cycles 1 (black), 100 (red) and 1000 (blue). The device was scanned between 1.5 and $-1.5 \mathrm{~V}$ at a scan rate of $50 \mathrm{mV} \mathrm{s}^{-1}$.

PEDOT:Tos. Final practical devices, which will be exposed to sunlight during daytime, may also benefit from the use of UV and oxygen protective encapsulation layers to prevent potential issues with photo-oxidation reactions. ${ }^{44}$

\section{Conclusions}

This report demonstrates lateral and vertical organic IR electrochromic devices based on the conductive polymer PEDOT:Tos. Due to its high conductivity ( $>1000 \mathrm{~S} \mathrm{~cm}^{-1}$ ), PEDOT:Tos could be used not only as an electrochromic material, but also as an electrode, enabling the development of the first metal-free flexible IR electrochromic device. The conductive polymer was encapsulated using polyethylene substrates, which was found to be suitable as an IR transparent material that also offers high flexibility. The use of a gel electrolyte promoted stability and a large electrochemical window, while maintaining suitable switching speeds. The flexible PEDOT devices were studied by UV-vis and FT-IR transmittance and reflectance spectroscopy, showing modulation over both the visible and IR regions. Thermal imaging of a lateral electrochromic device provided an effective temperature difference of almost $10{ }^{\circ} \mathrm{C}$ between the two redox states, which is almost one order of magnitude higher than previous IR electrochromic devices based on PEDOT. The vertical electrochromic device architecture produced slightly smaller temperature differences, yet still over $6{ }^{\circ} \mathrm{C}$ and with rapid switching of only a few seconds. A more detailed investigation of the vertical electrochromic devices showed that they require very low power consumption while also providing good optical memory, with retained contrast between the 'on' and 'off' states after at least 10 hours. The stability of the devices was good to up to approximately 100 cycles, as investigated by cyclic voltammetry, while more long-term stability $(\sim 1000$ cycles $)$ requires further optimization. These device specifications, along with their lightweight and simple design, highlight the strong potential of PEDOT:Tos for IR electrochromic applications, including for dynamic road signs with thermal signatures.

\section{Acknowledgements}

The authors acknowledge financial support from the Wenner-Gren Foundations, the Swedish Research Council, the Swedish Foundation for Strategic Research, the ÅForsk Foundation, Knut and Alice Wallenberg Foundation (Tail of the sun), Vinnova as part of the Swedish national innovation program SIO Graphene, and the Swedish Government Strategic Research Area in Materials Science on Functional Materials at Linköping University (Faculty Grant SFO-Mat-LiU No. 2009 00971).

\section{References}

1 C. Filloy, M. Omar and Y. Zhou, Sens. Rev., 2007, 27, 310-316. 2 C. Dai, Y. Zheng and $\mathrm{X}$. Li, Computer Vision and Image Understanding, 2007, 106, 288-299. 
3 O. David, N. S. Kopeika and B. Weizer, Appl. Opt., 2006, 45, 7248-7254.

4 W. H. Weber, T. Potter and A. Shaffer, US Pat., 20050206510 A1, 2005.

5 K. Owens and L. Matthies, Computer Vision Beyond the Visible Spectrum: Methods and Applications, 1999. (CVBVS '99) Proceedings. IEEE Workshop on, Fort Collins, CO, 1999, 59-68.

6 C. Y. Fang, C. S. Fuh, P. S. Yen, S. Cherng and S. W. Chen, Computer Vision and Image Understanding, 2004, 96, 237-268.

7 A. Halstuch and Y. Yitzhaky, Appl. Opt., 2008, 47, 4031-4036.

8 M. Bertozzi, A. Broggi, P. Grisleri, T. Graf and M. Meinecke, IEEE IV2003 Intelligent Vehicles Symposium. Proceedings (Cat. No.03TH8683), 2003, 662-667.

9 K. Bange and T. Gambke, Adv. Mater., 1990, 2, 10-16.

10 D. R. Rosseinsky and R. J. Mortimer, Adv. Mater., 2001, 13, 783-793.

11 C. M. Lampert, Sol. Energy Mater., 1984, 11, 1-27.

12 P. R. Somani and S. Radhakrishnan, Mater. Chem. Phys., 2003, 77, 117-133.

13 W. Lu, A. G. Fadeev, B. Qi and B. R. Mattes, J. Electrochem. Soc., 2004, 151, H33-H39.

14 D. Kumar and R. C. Sharma, Eur. Polym. J., 1998, 34, 1053-1060.

15 L. Groenendaal, F. Jonas, D. Freitag, H. Pielartzik and J. R. Reynolds, Adv. Mater., 2000, 12, 481-494.

16 R. Brooke, M. Fabretto, N. Vucaj, K. Zuber, E. Switalska, L. Reeks, P. Murphy and D. Evans, Smart Mater. Struct., 2015, 24, 035016.

17 H. Shin, S. Seo, C. Park, J. Na, M. Han and E. Kim, Energy Environ. Sci., 2016, 9, 117-122.

18 M. Fabretto, J.-P. Autere, D. Hoglinger, S. Field and P. Murphy, Thin Solid Films, 2011, 519, 2544-2549.

19 C. Louet, S. Cantin, J.-P. Dudon, P.-H. Aubert, F. Vidal and C. Chevrot, Sol. Energy Mater. Sol. Cells, 2015, 143, 141-151.

20 B. Kim, J. Koh, J. Park, C. Ahn, J. Ahn, J. Kim and S. Jeon, Nano Convergence, 2015, 2, 1-7.

21 A. P. Sandoval, M. F. Suárez-Herrera and J. M. Feliu, Beilstein J. Org. Chem., 2015, 11, 348-357.

22 B. Kim, H. Shin, T. Park, H. Lim and E. Kim, Adv. Mater., 2013, 25, 5483-5489.

23 C. Pozo-Gonzalo, D. Mecerreyes, J. A. Pomposo, M. Salsamendi, R. Marcilla, H. Grande, R. Vergaz, D. Barrios and J. M. SánchezPena, Sol. Energy Mater. Sol. Cells, 2008, 92, 101-106.

24 P. Chandrasekhar, B. J. Zay, G. C. Birur, S. Rawal, E. A. Pierson, L. Kauder and T. Swanson, Adv. Funct. Mater., 2002, 12, 95-103.
25 A. Bessiére, C. Marcel, M. Morcrette, J.-M. Tarascon, V. Lucas, B. Viana and N. Baffier, J. Appl. Phys., 2002, 91, 1589-1594.

26 J. Ouyang, C. W. Chu, F. C. Chen, Q. Xu and Y. Yang, Adv. Funct. Mater., 2005, 15, 203-208.

27 R. Brooke, P. Cottis, P. Talemi, M. Fabretto, P. Murphy and D. Evans, Prog. Mater. Sci., 2017, 86, 127-146.

28 M. V. Fabretto, D. R. Evans, M. Mueller, K. Zuber, P. HojatiTalemi, R. D. Short, G. G. Wallace and P. J. Murphy, Chem. Mater., 2012, 24, 3998-4003.

29 M. Mueller, M. Fabretto, D. Evans, P. Hojati-Talemi, C. Gruber and P. Murphy, Polymer, 2012, 53, 2146-2151.

30 B. Cho, K. S. Park, J. Baek, H. S. Oh, Y.-E. Koo Lee and M. M. Sung, Nano Lett., 2014, 14, 3321-3327.

31 O. Bubnova, Z. U. Khan, H. Wang, S. Braun, D. R. Evans, M. Fabretto, P. Hojati-Talemi, D. Dagnelund, J.-B. Arlin, Y. H. Geerts, S. Desbief, D. W. Breiby, J. W. Andreasen, R. Lazzaroni, W. M. Chen, I. Zozoulenko, M. Fahlman, P. J. Murphy, M. Berggren and X. Crispin, Nat. Mater., 2014, 13, 190-194.

32 D. Evans, M. Fabretto, M. Mueller, K. Zuber, R. Short and P. Murphy, J. Mater. Chem., 2012, 22, 14889-14895.

33 R. Brooke, D. Evans, M. Dienel, P. Hojati-Talemi, P. Murphy and M. Fabretto, J. Mater. Chem. C, 2013, 1, 3353-3358.

34 B. Winther-Jensen and K. West, Macromolecules, 2004, 37, 4538-4543.

35 J. Hwang, I. Schwendeman, B. Ihas, R. Clark, M. Cornick, M. Nikolou, A. Argun, J. Reynolds and D. Tanner, Phys. Rev. B: Condens. Matter Mater. Phys., 2011, 83, 195121.

36 M. Fabretto, K. Zuber, C. Hall and P. Murphy, Macromol. Rapid Commun., 2008, 29, 1403-1409.

37 W. Banks, M. Gordon and A. Sharples, Polymer, 1963, 4, 289-302.

38 A. J. H. Batting, T. G. Owen, M. Standberg, A. Sawatdee, X. Wang, A. Malmstrom, M. Nilsson and L. O. Hennerdal, US pat., 20120126931A1, 2012.

39 C. D. Reid and E. D. McAlister, J. Opt. Soc. Am., 1959, 49, 78-82. 40 J. Vila, P. Ginés, J. M. Pico, C. Franjo, E. Jiménez, L. M. Varela and O. Cabeza, Fluid Phase Equilib., 2006, 242, 141-146.

41 P. Heremans, G. H. Gelinck, R. Müller, K.-J. Baeg, D.-Y. Kim and Y.-Y. Noh, Chem. Mater., 2011, 23, 341-358.

42 D. Mecerreyes, R. Marcilla, E. Ochoteco, H. Grande, J. A. Pomposo, R. Vergaz and J. M. Sánchez Pena, Electrochim. Acta, 2004, 49, 3555-3559.

43 P. Andersson Ersman, J. Kawahara and M. Berggren, Org. Electron., 2013, 14, 3371-3378.

44 R. H. Bulloch and J. R. Reynolds, J. Mater. Chem. C, 2016, 4, 603-610. 